\title{
Adesão de biofilmes monoespécie de Streptococcus mutans e Candida albicans em diferentes superfícies de resinas compostas convencionais e bulk fill
}

\author{
Adhesion of monospecies biofilms from Streptococcus mutans and Candida \\ albicans to different surfaces of conventional composite resins and bulk fill
}

\author{
Arella Cristina Muniz BRITOa (D), Isis Morais BEZERRA ${ }^{a}$ (D), Maria Heloísa de Souza BORGES ${ }^{b}$ (D), \\ Rênnis de Oliveira da SILVA ${ }^{a}$ (i), Francisco Naldo GOMES FILHO ${ }^{b} \mathbb{B}^{(1)}$, \\ Leopoldina de Fátima Dantas de ALMEIDA** (1) \\ aUFPB - Universidade Federal da Paraíba, Programa de Pós-graduação em Odontologia, João Pessoa, PB, Brasil \\ bUFPB - Universidade Federal da Paraíba, Graduação em Odontologia, João Pessoa, PB, Brasil \\ 'UFPB - Universidade Federal da Paraíba, Departamento de Odontologia e Clínica Social, Programa de Pós-graduação \\ em Odontologia, João Pessoa, PB, Brasil
}

\begin{abstract}
Como citar: Brito ACM, Bezerra IM, Borges MHS, Silva RO, Gomes Filho FN, Almeida LFD. Adesão de biofilmes monoespécie de Streptococcus mutans e Candida albicans em diferentes superfícies de resinas compostas convencionais e bulk fill. Rev Odontol UNESP. 2020;49:e20200015. https://doi.org/10.1590/1807-2577.01520
\end{abstract}

\begin{abstract}
Resumo
Introdução: As resinas compostas são alternativas restauradoras, porém sua superfície pode favorecer o acúmulo de biofilme. Objetivo: Analisar in vitro a adesão de biofilmes de Streptococcus mutans (UA159) e Candida albicans (ATCC 90028) em superfícies de resinas compostas convencionais e bulk fill. Material e método: Foram utilizadas quatro marcas de resinas compostas e bulk fill: Aura Bulk Fill - SDI®; Premisa - Kerr®; Opallis- FGM®, e Filtek bulk fill flow - 3M®. Utilizou-se saliva artificial para formação da película salivar, por $60 \mathrm{~min}$ a $37^{\circ} \mathrm{C}$. 0 inóculo foi padronizado em $1 \times 10^{8}$ $\mathrm{UFC} / \mathrm{mL}$ para $S$. mutans e $1 \times 10^{6} \mathrm{UFC} / \mathrm{mL}$ para $C$. albicans. Os espécimes (n=8/grupo) foram acondicionados em placas de 24 poços, com BHI suplementado com sacarose para as bactérias, e RPMI 1640, para os fungos. A formação do biofilme foi avaliada considerando as unidades formadoras de colônia (UFC/mL).Os dados foram analisados por ANOVA e Tukey $(\mathrm{p}<0,05)$. Resultado: Para os biofilmes de $S$. mutans, não houve diferença significativa na contagem de UFC/mL entre os diferentes tipos de resina $(\mathrm{p}=0,119)$. Na contagem de UFC $/ \mathrm{mL}$ para biofilme de Candida, as médias variaram entre 7,78 e 8,34. Houve diferença significativa entre as marcas, especialmente entre as resinas convencionais e bulk fill. Conclusão: 0 presente estudo demonstra que não há diferença na adesão para biofilmes de $S$. mutans. Porém, há diferença na adesão da C. albicans na superfície de diferentes resinas compostas.
\end{abstract}

Descritores: Streptococcus mutans; Candida albicans; restauração dentária permanente; materiais dentários.

\begin{abstract}
Introduction: Composite resins are restorative alternatives, but their surface may favor the accumulation of biofilm. Objective: The aim of this study was to evaluate the in vitro adhesion of Streptococcus mutans (UA159) and Candida albicans (ATCC 90028) biofilms on the surface of conventional and bulk fill composites. Material and method: Four brands of conventional and bulk fill composites were used, Aura Bulk Fill - SDI@, Premisa - Kerr®, Opallis- FGM ${ }^{\circledR}$ and Filtek bulk fill flow - $3 \mathrm{M} \circledast$. Artificial saliva was used to form the salivary film for $60 \mathrm{~min}$ at $37^{\circ} \mathrm{C}$. The inoculum was standardized at $1 \times 108 \mathrm{CFU} / \mathrm{mL}$ for S. mutans and $1 \times 106 \mathrm{CFU} / \mathrm{mL}$ for C. albicans. The specimens ( $\mathrm{n}=8$ /group) were placed in 24-well plates, with BHI supplemented with sucrose for bacteria and RPMI 1640 for fungi. The biofilm formation was evaluated considering the colony forming units $(\mathrm{CFU} / \mathrm{mL})$. The data were analyzed by ANOVA and Tukey test $(\mathrm{p}<0.05)$. Result: For $S$. mutans biofilms, there was no significant difference in the $\mathrm{CFU} / \mathrm{mL}$ count between the different types of
\end{abstract}


composites ( $\mathrm{p}=0.119$ ). In the CFU / $\mathrm{mL}$ count for C.albicans biofilm, the CFU/mL ranged from 7.78 to 8.34. There was a significant difference between brands for Candida, especially between conventional and bulk fill composites. Conclusion: The present study demonstrates that there is no difference in adhesion for $S$. mutans biofilms. On the other hand, there is a difference in the adhesion of $C$. albicans to the surface of different composite resins.

Descriptors: Streptococcus mutans; Candida albicans; dental restoration, permanent; dental materials.

\section{INTRODUÇÃO}

O uso de resinas compostas para dentes anteriores e posteriores tem aumentado nos últimos anos devido às suas propriedades estéticas ${ }^{1,2}$. Há uma variedade de opções disponíveis, que apresentam diferenças na composição e no tamanho da partícula, por exemplo. Podem ser desde as resinas convencionais, utilizadas por meio da técnica incremental, até as resinas bulk fill, que surgiram como uma alternativa para minimizar desvantagens, como a contração de polimerização $0^{3-5}$.

Entretanto, as resinas compostas estão mais susceptíveis a adesão de microrganismos e formação de biofilme, e a uma maior frequência de substituição, quando comparadas a um material restaurador, como o amálgama, por exemplo, o que pode interferir na longevidade das restaurações ${ }^{6-8}$.

Microrganismos, como o Streptococcus mutans, estão associados ao desenvolvimento da cárie dentária, devido a sua capacidade de adesão e formação de biofilme. Estas características podem estar associadas ao surgimento de cáries secundárias em elementos restaurados com materiais resinosos, comprometendo estas restaurações ao longo dos anos ${ }^{9-12}$.

Outro microrganismo que apresenta propriedade de adesão a superfícies dentárias e resinas compostas é a Candida albicans, que também está presente na microbiota da cárie dentária13. Além disso, esse microrganismo está comumente classificado como o microrganismo mais relacionado à candidíase oral ${ }^{14}$, o que pode vir a ser um problema de saúde pública, principalmente para pacientes imunocomprometidos e usuários de próteses, pois a adesão de biofilmes a essa superfície pode funcionar como um reservatório fúngico ${ }^{15-}$ 17.

Tendo em vista que a propriedade de adesão microbiológica também é importante para a correta indicação desses materiais restauradores, o objetivo do presente estudo foi comparar, in vitro, a suscetibilidade de quatro materiais restauradores (duas resinas compostas convencionais, uma resina bulk fill e outra bulk fill flow) a biofilmes monoespécie separados de Streptococcus mutans e Candida albicans.

A hipótese testada no presente estudo foi se a adesão microbiana apresenta diferenças dependendo da resina composta utilizada, tanto para biofilmes monoespécie de $S$. mutans quanto de C. albicans.

\section{MATERIAL E MÉTODO}

\section{Preparo dos Espécimes}

Foram utilizadas quatro marcas diferentes de resinas compostas e bulk fill disponíveis no mercado: Aura Bulk Fill - SDI $\AA$; Premisa - Kerr®; Opallis- FGM®, e Filtek bulk fill flow - 3M®. Todas as informações relevantes e as características dos compósitos de resinas utilizadas estão apresentadas no Quadro 1. 
Quadro 1. Caracterização das resinas compostas

\begin{tabular}{|c|c|c|c|c|c|}
\hline $\begin{array}{c}\text { Material } \\
\text { Restaurador }\end{array}$ & Tipo & Partícula & Composição & Fabricante & Lote \\
\hline Aura & \begin{tabular}{|} 
Ultra High \\
Density \\
(UHD) glass \\
filler \\
\end{tabular} & Nano-híbrida & Não disponível & $\begin{array}{l}\text { Southern Dental } \\
\text { Industries (SDI) }\end{array}$ & 150710 \\
\hline Opallis & $\begin{array}{c}\text { Resina } \\
\text { composta } \\
\text { convencional }\end{array}$ & Micro-híbrida & $\begin{array}{l}\text { Bis (GMA), Bis (EMA), UDMA e } \\
\text { TEGDMA, vidro de Bário- } \\
\text { Alumino silicato silanizados e } \\
\text { nanopartículas de dióxido de } \\
\text { silício, canforquinona como } \\
\text { fotoiniciador, aceleradores, } \\
\text { estabilizantes e pigmentos. }\end{array}$ & Fgm, SC, Brasil & 030516 \\
\hline Premisa & $\begin{array}{c}\text { Resina } \\
\text { composta } \\
\text { convencional }\end{array}$ & Nano-híbrida & $\begin{array}{c}\text { Uncured methacrylate ester } \\
\text { monomers (20-35\%), inert } \\
\text { mineral fillers, activators and } \\
\text { stabilizers } \\
\end{array}$ & $\begin{array}{c}\text { Kerr Corporation } \\
\text { Dental, EUA }\end{array}$ & 5842523 \\
\hline $\begin{array}{l}\text { Filtek Bulk fill } \\
\text { flow }\end{array}$ & $\begin{array}{l}\text { Resina em } \\
\text { Bulk de } \\
\text { baixa } \\
\text { contração } \\
\text { flow }\end{array}$ & Micro-híbrida & $\begin{array}{l}\text { Cerâmica silanizada tratada; } \\
\text { diuretano dimetacrilato } \\
\text { (UDMA), dimetacrilato } \\
\text { substituída; bisfenol A } \\
\text { polietilenoglicol diéter } \\
\text { dimetacrilato (BISEMA); } \\
\text { fluoreto de itérbio, bisfenol A } \\
\text { di-(2-hidroxi-propoxi) } \\
\text { dimetacrilato de } \\
\text { trietilenoglicol (TEGDMA) e } \\
\text { etil 4-dimetilaminobenzoato }\end{array}$ & 3M Company & 1727300140 \\
\hline
\end{tabular}

Para cada marca, foram confeccionados espécimes de 7,5 $\times$ 7,5 $\times 3 \mathrm{~mm}(\mathrm{n}=8)$, em um molde plástico, de acordo com instruções dos fabricantes, e fotoativadas por luz de LED (Emitter C, Schuster, $1250 \mathrm{~mW} / \mathrm{cm}^{2} ; 2 \mathrm{~cm}$ distância da ponta) durante 40 segundos. Em seguida, as amostras foram removidas dos moldes e polidas com lixa d'água, médias e finas, e com discos abrasivos (TDV ${ }^{\circledR}, \mathrm{SC}$, Brasil), utilizando uma peça de mão de baixa rotação ${ }^{15}$.

\section{Cálculo Amostral}

0 cálculo do número de amostras foi realizado considerando o poder $(\beta)$ de $80 \%$ e alta magnitude de efeito por meio do método de diferença bruta entre as médias $(8,34 \pm 0,15$ e 7,78 \pm $0,16)$. Assim, foram incluídos oito espécimes por grupo a partir de estudo prévio.

\section{Desenvolvimento dos Biofilmes}

Os espécimes de resina composta foram aderidos em um fio ortodôntico ํㅡ 07 (Morelli ${ }^{\circledR}$, SP, Brasil) em formato de $\mathrm{Z}$ e este, com auxílio de cera utilidade, fixado à tampa de uma placa de cultura de 24 compartimentos (KASVI ${ }^{\circledR}, \mathrm{PR}$, Brasil). 0 desenho experimental e os detalhes da disposição dos espécimes de resina podem ser observados na Figura 1. 


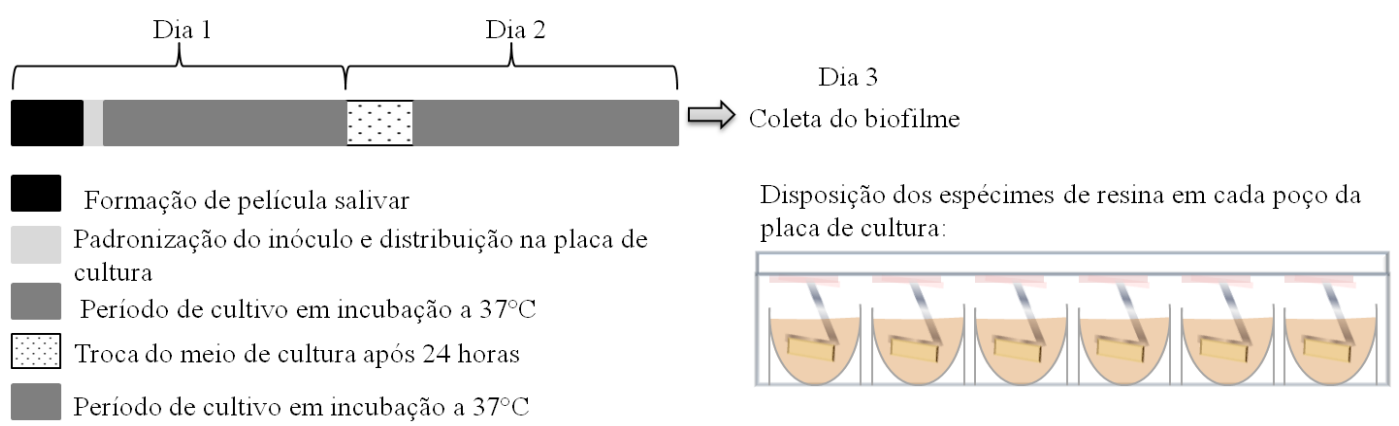

Figura 1. Desenho experimental e detalhes do modelo de biofilme, e disposição dos espécimes na placa de cultura.

Assim, os espécimes foram dispostos de maneira a ficarem suspensos de forma perpendicular ao fundo do compartimento e imersos no meio de cultura. Esta manobra permitiu que os mesmos não tocassem o acrílico da placa, impedindo a adesão dos microrganismos apenas por deposição (Figura 1). Esta proposta foi adaptada de um modelo de biofilme referenciado na literatura ${ }^{18}$. Após a preparação, os espécimes foram esterilizados com óxido etileno.

Inicialmente, a película salivar foi formada com saliva artificial (carboximetil 1\%, cloreto de sódio $0,0084 \%$, cloreto de potássio $0,12 \%$, fosfato de potássio monobásica $0,0342 \%$, cloreto de cálcio 0,0146\%, cloreto de magnésio 0,0052\%, Farmácia Farmafórmula, Farmácia de Manipulação, João Pessoa-PB, Brasil). Foram adicionados $2 \mathrm{~mL}$ de saliva artificial por espécime em cada compartimento. Em seguida, a placa de cultura foi incubada por 60 min a $37{ }^{\circ} \mathrm{C}$. Posteriormente, os inóculos padrões de ambos os microrganismos foram inseridos nas placas de cultura, como descrito a seguir.

\section{Biofilme Monoespécie de Streptococcus mutans}

Após a adesão da película salivar nos espécimes de resina, adicionou-se à placa de cultura o inóculo padronizado com a cepa de referência Streptococcus mutans (UA159), na densidade celular de $1 \times 10^{8} \mathrm{UFC} / \mathrm{mL}$. Dessa maneira, foi adicionado $1 \mathrm{~mL}$ de meio de cultura BHI (HIMEDIA ${ }^{\circledR}$, Mumbai, Índia) suplementado com $1 \%$ de sacarose, em cada compartimento das placas de 24 poços (KASVI ${ }^{\circledR}, \mathrm{PR}$, Brasil), mantendo-as incubadas por $48 \mathrm{~h}$ a $37^{\circ} \mathrm{C}$ em microaerofilia, com troca do meio de cultura a cada $24 \mathrm{~h}$. Após esse período de cultivo, os espécimes foram coletados para a análise das Unidades Formadoras de Colônia (UFC).

\section{Biofilme Monoespécie de Candida albicans}

Em outro experimento independente, foi adicionado à placa de cultura com os espécimes de resinas com a película salivar já formada, o inóculo padronizado de Candida albicans (ATCC 90028) com densidade celular de $1 \times 10^{6} \mathrm{UFC} / \mathrm{mL}$. Foi adicionado $1 \mathrm{~mL} /$ poço com meio de cultura RPMI 1640 (Inlab Diagnóstica, SP, Brasil). A placa de cultura foi incubada a $37^{\circ} \mathrm{C}$ totalizando um período de cultivo de $48 \mathrm{~h}$, com trocas do meio de cultura a cada $24 \mathrm{~h}$.

Os espécimes foram acondicionados em placas de 24 poços (KASVI ${ }^{\circledR}, \mathrm{PR}$, Brasil), adicionandose $1 \mathrm{~mL} /$ poço com incubação por $48 \mathrm{~h}$ a $37^{\circ} \mathrm{C}$ em estufa (Callmex ${ }^{\circledR}, \mathrm{SC}$, Brasil), com troca do meio de cultura a cada $24 \mathrm{~h}$. 


\section{Unidades Formadoras de Colônia}

Na análise das Unidades Formadoras de Colônia (UFC), os espécimes com os biofilmes desenvolvidos em sua superfície foram removidos da estrutura metálica e adicionados a microtubos com $1 \mathrm{~mL}$ solução salina a 0,9\% previamente adicionado. Em seguida, os microtubos foram submetidos à agitação no vórtex durante 30 segundos para desprendimento do biofilme.

Foram coletados $200 \mu \mathrm{L}$ dos microtubos para realizar as microdiluições nas placas de 96 poços (KASVI ${ }^{\circledR}, \mathrm{PR}$, Brasil). Em seguida, as concentrações foram plaqueadas por meio da técnica da gota, $10 \mu \mathrm{L}$ em triplicata por concentração, para posterior incubação em estufa (Callmex ${ }^{\circledR}, \mathrm{SC}$, Brasil) por 24 horas.

Em relação ao biofilme monoespécie de Streptococcus mutans, as unidades formadoras de colônia foram analisadas em Mitis Salivarius Agar (MSA) (Synth KASVI ${ }^{\circledR}$, PR, Brasil), SP, Brasil) suplementado com sacarose. Para a formação do biofilme monoespécie de Candida albicans, foram avaliadas as unidades formadoras de colônia (UFC/mL) em placas de Agar Sabouraud Dextrose (KASVI ${ }^{\circledR}, \mathrm{PR}$, Brasil).

\section{Análise dos Dados}

O cultivo dos biofilmes foi realizado de modo independente, em dois momentos distintos. Inicialmente, para o cultivo do biofilme de $S$. mutans, e em outro momento, para o cultivo de $C$. albicans. Foi realizada a triplicata nas placas de cultura para a contagem de UFC.

O software SPSS versão 20 (IBM, Chicago, IL) foi utilizado na análise estatística. As diferenças entre os espécimes de resina foram observadas por meio da análise de variância a critério fixo (ANOVA one-way) e do teste de Tukey, para determinar quais médias foram significativamente diferentes a $5 \%$ de significância.

\section{RESULTADO}

Na Tabela 1, é possível observar as médias dos valores encontrados para a contagem de UFC/mL para $S$. mutans, na diluição de $10^{-5}$. Na tabela, é indicada a marca de resina utilizada e seus respectivos valores. Destaca-se que as médias variaram de 8,32 a 8,52 e que não houve diferença significativa entre nenhuma das marcas avaliadas $(\mathrm{p}=0,119)$, o que foi observado ao se aplicar o teste ANOVA/one way.

Tabela 1. Contagem de UFC/mL para biofilmes de Streptococcus mutans em diferentes resinas compostas (Média, Desvio Padrão e ANOVA)

\begin{tabular}{cccccc}
\hline Resina Composta & $\begin{array}{c}\text { Soma dos } \\
\text { quadrados }\end{array}$ & GL & Teste F & UFC/mL (Média e DP) & Significância \\
\hline Aura bulk fill & & & & $8,33 \pm 0,20$ & \\
Opallis & \multirow{2}{*}{29} & 2,133 & $8,36 \pm 0,20$ & 0,119 \\
Premisa & & & & $8,52 \pm 0,08$ & \\
Filtek bulk fill & & & & $8,32 \pm 0,21$ & \\
\hline
\end{tabular}

Os valores não foram estatisticamente diferentes ( $p>0,05)$ (ANOVA) UFC/mL: Unidades Formadoras de Colônias por mililitro; DP: desvio padrão; ANOVA: Análise de variância; GL: graus de liberdade; Teste F: teste de Snedecor; SPSS versão 20 (IBM, Chicago, IL); SPSS versão 20 (IBM, Chicago, IL).

Para a análise de UFC/mL para biofilmes de C. albicans, na diluição de $10^{-5}$, as médias variaram de 7,78 a 8,34. A partir do teste ANOVA/one way, foi observado que houve diferença entre os grupos. Posteriormente, foi realizado o Teste de Tukey, para identificar tais diferenças. 
Dessa maneira, verificou-se maior número de células aderidas à resina Filtek bulk fill $(8,34 \pm 0,15)$, devido à maior média de contagem de UFC $/ \mathrm{mL}$. A menor contagem foi determinada para a resina Aura bulk fill $(7,78 \pm 0,16)$, havendo assim diferenças significativas entre ambas $(\mathrm{p}=0,006)$ (Tabela 2$)$.

Tabela 2. Contagem de UFC/mL para biofilmes de Candida albicans em diferentes resinas compostas (Média, Desvio Padrão e ANOVA)

\begin{tabular}{ccccc}
\hline Resina Composta & $\begin{array}{c}\text { Soma dos } \\
\text { quadrados }\end{array}$ & GL & Teste F & $\begin{array}{c}\text { UFC/mL (Média e } \\
\text { DP) }\end{array}$ \\
\hline Aura bulk fill & & & & $7,78 \pm 0,16^{\mathrm{a}}$ \\
Opallis & \multirow{2}{*}{29} & 5,693 & $7,91 \pm 0,92^{\mathrm{a}, \mathrm{b}}$ \\
Premisa & & & $7,85 \pm 0,71^{\mathrm{a}}$ \\
Filtek bulk fill & & & $8,34 \pm 0,15^{\mathrm{b}}$ \\
\hline
\end{tabular}

Os valores foram estatisticamente diferentes $(p \leq 0,05)$, o que pode ser observado verticalmente, em que letras diferentes indicam diferenças significativas entre as diferentes resinas compostas ( $\mathrm{p}<0,05)$ (ANOVA one-way/Tukey); UFC/mL: Unidades formadoras de colônias por mililitro; DP: desvio padrão; ANOVA: Análise de variância; GL: graus de liberdade; Teste F: teste de Snedecor; SPSS versão 20 (IBM, Chicago, IL).

A resina bulk fill da marca Aura não apresentou diferença significativa quando comparada às resinas do tipo convencionais Opallis $(p=0,845)$ e Premisa $(p=0,957)$. Também não houve diferença entre as marcas Opallis e Premissa $(p=0,987)$. Já para a marca de resina bulk fill flow Filtek, houve diferença significativa entre as resinas Aura $(p=0,006)$ e Premisa $(p=0,019)$; entretanto, não houve diferença quando comparada à resina convencional Opallis $(\mathrm{p}=0,051)$. As médias e os desvios padrão podem ser observados na Tabela 2 .

\section{DISCUSSÃO}

O presente estudo buscou avaliar o efeito da adesão de microrganismos em materiais restauradores de quatros resinas compostas diferentes em relação a biofilmes de $S$. mutans e C. albicans, por meio da contagem da UFC/mL, um método confiável que tem sido utilizado para estimar a população de microrganismos ${ }^{19}$.

A literatura tem demonstrado que a adesão microbiológica e o desenvolvimento de biofilmes estão relacionados à natureza do material e às características de sua superfície, incluindo propriedades, como composição química, liberação de íons e rugosidade de superfície ${ }^{20}$. Além disso, o tipo da matriz encontrada em resinas compostas pode influenciar o potencial de adesão do biofilme ${ }^{17}$ a depender do método de cultivo celular.

A adesão de $S$. mutans a resinas compostas foi determinada em estudo prévio, em que, para o S. mutans, especificamente, não houve diferença nas taxas de adesão bacterianas em diferentes composições de resinas compostas, inclusive entre resinas convencionais e bulk fill. As resinas também foram comparadas em relação a diferentes tamanhos de partículas e não foram observadas diferenças ${ }^{21}$. Porém, no referido estudo, os espécimes não foram submetidos à formação de película salivar e o tempo de desenvolvimento dos microrganismos foi apenas de quatro horas.

No presente estudo, também não foram encontradas diferenças significativas entre as marcas de resinas utilizadas, mesmo ao se desenvolver um biofilme com saliva artificial, cultivado por 48 horas. Uma possível explicação para esse fato ao polimento dos espécimes, que pode interferir nas taxas de adesão; porém, neste estudo, todos os espécimes foram polidos da mesma maneira ${ }^{9,12}$. A superfície pode exercer uma influência maior na adesão microbiana do que outros fatores, como a composição, por exemplo. Todavia, não pode ser estabelecida uma relação direta, 
visto que superfície e adesão bacteriana não foram variáveis correlacionadas na presente pesquisa.

Em relação ao biofilme monoespécie de $C$. albicans, houve diferença significativa entre os diferentes tipos de resinas investigadas. A resina bulk fill Filtek Flow apresentou a maior taxa de $\mathrm{UFC} / \mathrm{mL}$, seguida da Opallis. Ambas apresentam em comum a natureza micro-híbrida da partícula, o que pode ser uma possível explicação para uma maior taxa de adesão a essas superfícies, para esse tipo de biofilme.

Há uma escassez de estudos que compararam a adesão de C. albicans em resinas compostas. 0 estudo publicado por Beldüz et al.15, que, apesar de utilizar método, tempo e meio de cultura diferentes, avaliou a formação de biofilme do referido microrganismo em diferentes materiais restauradores por meio do teste colorimétrico tetrazolium XTT [2,3-bis(2-methoxy-4-nitro-5sulfophenyl)-2H-tetrazolium-5-carboxanilide].

Dentre os materiais utilizados no estudo, a resina composta nano-híbrida apresentou maior adesão da C. albicans, seguida pela resina composta modificada por ionômero de vidro e por outra resina nano-híbrida, quando comparados aos materiais amálgama e ionômero de vidro. Os autores propuseram que o material influenciou na aderência da Candida e também ressaltaram a importância da escolha criteriosa desses materiais, principalmente para pacientes imunocomprometidos ${ }^{15}$.

Além disso, destacamos a necessidade de mais estudos que avaliem a adesão de $C$. albicans a diferentes resinas compostas, tendo em vista que esse microrganismo consegue aderir a tal substrato e também está presente em biofilmes cariogênicos, como já foi reportado na literatura, inclusive em cáries em dentina ${ }^{13,22}$, o que também pode estar relacionado a casos de cáries secundárias.

Os microrganismos utilizados no presente ensaio foram incluídos devido à estreita relação destes na formação do biofilme cariogênico, considerando a presença do $S$. mutans e $C$. albicans. Além disto, este fungo é agente causador de candidíase oral, sendo também um componente da microbiota de indivíduos sem agravos bucais $22-24$.

Porém, em um estudo prévio semelhante, foi demonstrado que a adesão microbiana de outras espécies também pode ser influenciada pela rugosidade do material. Colonizadores iniciais, como os estreptococos orais, assim como bactérias gram-negativas de colonização tardia, podem apresentar diferentes taxas de adesão, as quais variam de acordo com a rugosidade das resinas compostas utilizadas. Assim, o acabamento periódico da rugosidade da superfície é um fator que deve ser considerado para minimizar a adesão de microrganismos às resinas compostas ${ }^{25}$.

Além disso, o tipo e a natureza da restauração também influenciam na capacidade de os microrganismos aderirem ao material. Por exemplo, a partir da contagem de células bacterianas por unidade de área, é observado que restaurações indiretas são menos propensas à adesão de biofilme bacterianos quando comparadas às restaurações de resinas compostas diretas, o que pode estar relacionado a rugosidade e topografia das restaurações ${ }^{26}$.

Dessa maneira, a partir dos resultados encontrados, podemos sugerir que além da composição e polimento, a metodologia aplicada e o tipo de biofilme também são fatores que interferem na adesão ao substrato e isto também é influenciado pelo tipo de microorganismo, visto que, no presente estudo, quanto à adesão do biofilme de C. albicans, observaram-se diferenças entre os grupos, e em relação ao biofilme de $S$. mutans, não houve diferenças significativas.

Entretanto, por se tratar de uma pesquisa in vitro, o presente estudo apresenta algumas limitações, pois foi realizado sob condições laboratoriais controladas que não podem ser extrapoladas diretamente para o contexto clínico, uma vez que não houve simulação de outros mecanismos de proteção da cavidade oral, como a presença de imunoglobulinas e a interação multiespécie dos biofilmes. 
Por outro lado, por se tratar de estudo primário, fornece os dados de maneira controlada, de acordo com objetivo do estudo, o que evita que demais variáveis interfiram nos resultados encontrados. Dessa maneira, outros estudos precisam ser realizados, que comparem a susceptibilidade das resinas a esses microrganismos, correlacionando também outros fatores, como composição e polimento da superfície, por exemplo, para a correta indicação desse material nas restaurações.

\section{CONCLUSÃO}

De acordo com o presente estudo, há diferença na adesão da $C$. albicans na superfície de diferentes marcas de resinas compostas, inclusive entre resinas compostas convencionais e bulk fill. Entretanto, não houve diferença nessa adesão para biofilmes de $S$. mutans. Destaca-se a necessidade de mais estudos que possam avaliar essas propriedades microbiológicas em diferentes substratos de resinas compostas.

\section{AGRADECIMENTOS}

Os autores agradecem ao Conselho Nacional de Desenvolvimento Científico e Tecnológico (CNPq) e à Coordenação de Aperfeiçoamento de Pessoal de Nível Superior (CAPES) pela concessão de Bolsa de mestrado.

\section{REFERÊNCIAS}

1. Rasines Alcaraz MG, Veitz-Keenan A, Sahrmann P, Schmidlin PR, Davis D, Iheozor-Ejiofor Z. Direct composite resin fillings versus amalgam fillings for permanent or adult posterior teeth. Cochrane Database Syst Rev. 2014 Mar;(3):CD005620. http://dx.doi.org/10.1002/14651858.CD005620.pub2. PMid:24683067.

2. Cazzaniga G, Ottobelli M, Ionescu AC, Paolone G, Gherlone E, Ferracane JL, et al. In vitro biofilm formation on resin-based composites after different finishing and polishing procedures. J Dent. 2017 Dec;67:43-52. http://dx.doi.org/10.1016/j.jdent.2017.07.012. PMid:28750776.

3. Bayraktar Y, Ercan E, Hamidi MM, Çolak H. One-year clinical evaluation of different types of bulk-fill composites. J Investig Clin Dent. 2017 May;8(2):e12210. http://dx.doi.org/10.1111/jicd.12210. PMid:26800647.

4. Demarco FF, Corrêa MB, Cenci MS, Moraes RR, Opdam NJM. Longevity of posterior composite restorations: not only a matter of materials. Dent Mater. 2012 Jan;28(1):87-101. http://dx.doi.org/10.1016/j.dental.2011.09.003. PMid:22192253.

5. El-Damanhoury H, Platt J. Polymerization shrinkage stress kinetics and related properties of bulk-fill resin composites. Oper Dent. 2014 Jul-Aug;39(4):374-82. http://dx.doi.org/10.2341/13-017-L. PMid:23865582.

6. Fúcio SB, Carvalho FG, Sobrinho LC, Sinhoreti MA, Puppin-Rontani RM. The influence of 30-day-old Streptococcus mutans biofilm on the surface of esthetic restorative materials - an in vitro study. J Dent. 2008 0ct;36(10):833-9. http://dx.doi.org/10.1016/j.jdent.2008.06.002. PMid:18621456.

7. Spencer P, Ye Q, Misra A, Goncalves SEP, Laurence JS. Proteins, pathogens, and failure at the compositetooth interface. J Dent Res. 2014 Dec;93(12):1243-9. http://dx.doi.org/10.1177/0022034514550039. PMid:25190266.

8. Zhang N, Melo MAS, Weir MD, Reynolds MA, Bai Y, Xu HHK. Do dental resin composites accumulate more oral biofilms and plaque than amalgam and glass ionomer materials? Materials (Basel). 2016 Nov;9(11):888. http://dx.doi.org/10.3390/ma9110888. PMid:28774007. 
9. Ono M, Nikaido T, Ikeda M, Imai S, Hanada N, Tagami J, et al. Surface properties of resin composite materials relative to biofilm formation. Dent Mater J. 2007 Sep;26(5):613-22. http://dx.doi.org/10.4012/dmj.26.613. PMid:18203458.

10. Delaviz Y, Finer Y, Santerre JP. Biodegradation of resin composites and adhesives by oral bacteria and saliva: a rationale for new material designs that consider the clinical environment and treatment challenges. Dent Mater. 2014 Jan;30(1):16-32. http://dx.doi.org/10.1016/j.dental.2013.08.201. PMid:24113132.

11. Salli KM, Ouwehand AC. The use of in vitro model systems to study dental biofilms associated with caries: a short review. J Oral Microbiol. 2015 Mar;7(1):26149. http://dx.doi.org/10.3402/jom.v7.26149. PMid:25740099.

12. Ionescu AC, Cazzaniga G, Ottobelli M, Ferracane JL, Paolone G, Brambilla E. In vitro biofilm formation on resin-based composites cured under different surface conditions. J Dent. 2018 Oct;77:78-86. http://dx.doi.org/10.1016/j.jdent.2018.07.012. PMid:30030124.

13. Dige I, Nyvad B. Candida species in intact in vivo biofilm from carious lesions. Arch Oral Biol. 2019 May;101:142-6. http://dx.doi.org/10.1016/j.archoralbio.2019.03.017. PMid:30933902.

14. Coronado-Castellote L, Jiménez-Soriano Y. Clinical and microbiological diagnosis of oral candidiasis. J Clin Exp Dent. 2013 Dec;5(5):e279-86. http://dx.doi.org/10.4317/jced.51242. PMid:24455095.

15. Beldüz N, Kamburoğlu A, Yıllmaz Y, Tosun İ, Beldüz M, Kara C. Evaluation of Candida albicans biofilm formation on various dental restorative material surfaces. Niger J Clin Pract. 2017 Mar;20(3):355-60. http://dx.doi.org/10.4103/1119-3077.198388. PMid:28256492.

16. Maza JL, Elguezabal N, Prado C, Ellacuría J, Soler I, Pontón J. Candida albicans adherence to resincomposite restorative dental material: influence of whole human saliva. Oral Surg Oral Med Oral Pathol Oral Radiol Endod. 2002 Nov;94(5):589-92. http://dx.doi.org/10.1067/moe.2002.126024. PMid:12424453.

17. Bürgers R, Schneider-Brachert W, Rosentritt M, Handel G, Hahnel S. Candida albicans adhesion to composite resin materials. Clin Oral Investig. 2009 Sep;13(3):293-9. http://dx.doi.org/10.1007/s00784-008-0226-4. PMid:18810508.

18. Fernández CE, Tenuta LMA, Cury JA. Validation of a cariogenic biofilm model to evaluate the effect of fluoride on enamel and root dentine demineralization. PLoS One. 2016 Jan;11(1):e0146478. http://dx.doi.org/10.1371/journal.pone.0146478. PMid:26731743.

19. Sieuwerts S, de Bok FA, Mols E, de vos WM, Vlieg JE. A simple and fast method for determining colony forming units. Lett Appl Microbiol. 2008 Oct;47(4):275-8. http://dx.doi.org/10.1111/j.1472765X.2008.02417.x. PMid:18778376.

20. Hao Y, Huang X, Zhou X, Li M, Ren B, Peng X, et al. Influence of dental prosthesis and restorative materials interface on oral biofilms. Int J Mol Sci. 2018 Oct;19(10):3157. http://dx.doi.org/10.3390/ijms19103157. PMid:30322190.

21. Motevasselian F, Zibafar E, Yassini E, Mirzaei M, Pourmirhoseni N. Adherence of Streptococcus mutans to microhybrid and nanohybrid resin composites and dental amalgam: an in vitro study. J Dent (Tehran). 2017 Nov;14(6):337-43. PMid:29942328.

22. Pereira D, Seneviratne CJ, Koga-Ito CY, Samaranayake LP. Is the oral fungal pathogen Candida albicans a cariogen? Oral Dis. 2018 May;24(4):518-26. http://dx.doi.org/10.1111/odi.12691. PMid:28513096.

23. Ellepola K, Liu Y, Cao T, Koo H, Seneviratne CJ. Bacterial GtfB augments Candida albicans accumulation in cross-kingdom biofilms. J Dent Res. 2017 Sep;96(10):1129-35. http://dx.doi.org/10.1177/0022034517714414. PMid:28605597.

24. Faustova MO, Ananieva MM, Basarab YO, Dobrobolska OV, Vovk IM, Loban' GA. Bacterial factors of cariogenicity (literature review). Wiad Lek. 2018;71(2 pt 2):378-82. PMid:29786589. 
25. Park J-W, An J-S, Lim WH, Lim B-S, Ahn S-J. Microbial changes in biofilms on composite resins with different surface roughness: an in vitro study with a multispecies biofilm model. J Prosthet Dent. 2019 Nov;122(5):493.e1-e8. https://doi.org/10.1016/j.prosdent.2019.08.009. PMid: 31648793.

26. Derchi G, Vano M, Barone A, Covani U, Diaspro A, Salerno M. Bacterial adhesion on direct and indirect dental restorative composite resins: an in vitro study on a natural biofilm. J Prosthet Dent. 2017 May;117(5):669-76. http://dx.doi.org/10.1016/j.prosdent.2016.08.022. PMid:27881324.

\section{CONFLITOS DE INTERESSE}

Os autores declaram não haver conflitos de interesse.

\section{*AUTOR PARA CORRESPONDÊNCIA}

Leopoldina de Fátima Dantas de Almeida, UFPB - Universidade Federal da Paraíba, Campus I, Castelo Branco, 58051-900 João Pessoa, PB, Brasil, e-mail: leopoldinalmeida@hotmail.com

Recebido: Março 5, 2020

Aprovado: Agosto 11, 2020 